\title{
Generation of Neural Cells by Direct Cellular Reprogramming
}

\section{Journal of \\ Transplantation \& Stem Cell Biology}

Keywords: Lineage conversion; Reprogramming; Neurons; Neural stem cells; G lial cells; Fibroblasts

\begin{abstract}
Cellular reprogramming is a new technique that is cumently explored for its potential to manufacture human cell types in vitro. Direct cellular reprogramming allows the direct conversion of cells into a target cell type of interest. This approach is of particular interest for cells of the central nervous system, which are notoriously difficult to obtain. Experiments in which cells were converted from an easily accessible starting cell to a different cell type across lineage boundaries have not only demonstrated an unexpected level of cellular plasticity of differentiated somatic cells, they also promise faster and more convenient manufacturing routes for various neura cell types. Renewable sources of human neurons and glia would be highly desirable for neurobiological studies, the development of cell replacement therapies to enhance regeneration in the CNS, disease modelling and drug-and phamacology testing. Here we review the existing literature investigating the generation of the principle cell types of the central nervous system: neurons and glia. We highlight some of the technical challenges that need to be overcome in order to realize the full potential of lineage reprogramming technologies.
\end{abstract}

\section{Introduction}

Recent advances in the field of stem cell research promise unprecedented opportunities for the study and potentially the treatment of a variety of disabling neurological diseases.

Human neural tissue is scarcely available and the isolated cells are difficult to expand. With the establishment of human embryonic stem cell (ESC) lines [1] an expandable source of human cells has emerged from which, in theory, all cells of the human organism including neural cells could be produced. The revolutionary discovery of induced pluripotent stem cells (iPSCs) [2], has enabled the generation of cells specific to human individuals. IPS technology thus allows the in vitro manufacture of patient-specific cells that ultimately may be used for autologous cell transplantation. However, the transplantation of iPSC-derived cells is only one of many potential biomedical applications. More immediately, iPS technology also promises to overcome some of the limitations inherent to animal models, which have formed the basis for neurological research until now. A reliable and consistent manufacture of human cell types specific to an individual would allow the modelling of human tissues and even the study of diseases "in a dish". Such culture systems may revolutionise the study of disease pathophysiology, pharmacological screening, and drug toxicology testing in the future.

However, until the potential of iPS technology can be realized many practical hurdles still need to be overcome. For example, the generation of standardised human iPSCs remains challenging and the differentiation protocols currently available for generating human neural cell-types are time-consuming and often do not meet the needs for large scale applications. The yield and the purity of the cells produced is often inconsistent and the differentiated cells obtained do not always reach the desired levels of cellular maturity.

\section{Matthias Pawlowski and Mark R Kotter*}

Anne McLaren Laboratory for Regenerative Medicine, MRC and Wellcome Trust Stem Cell Institute \& Department of Clinical Neurosciences, University of Cambridge, UK

Address for Correspondence

Mark R Kotter, MD, PhD, Anne McLaren Laboratory for Regenerative Medicine, MRC and Wellcome Trust Stem Cell Institute \& Department of Clinical Neurosciences, University of Cambridge, West Forvie Building, Robinson Way, CB2 0SZ Cambridge, UK, E-mail: mrk25@cam.ac.uk

Copyright: ( $\odot 2013$ Pawlowski M, et al. This is an open access article distributed under the Creative Commons Attribution License, which permits unrestricted use, distribution, and reproduction in any medium, provided the original work is properly cited.

Submission: 31 May 2013

Accepted: 16 July 2013

Published: 18 July 2013

More recently, the direct conversion of cells across major lineage boundaries emerged as a new strategy for the generation of neural cell types. This approach allows the transformation of easily available somatic cell types (typically fibroblasts) directly (i.e. without transition via a pluripotent intermediate) into a defined other cell type $[3,4]$ (Figure 1). Direct cellular reprogramming promises to overcome some of the problems associated with pluripotent stem cells and conventional directed differentiation protocols. In this review we provide a brief overview of recent advances in direct cellular reprogramming into CNS cell types and discuss the technical limitations of current reprogramming techniques. Overcoming these hurdles is essential for further progress in this exciting and rapidly developing field.

\section{Lineage Reprogramming: Conversion of Cells across Cell-lineage Boundaries}

The ability to reprogram cells across major germ-layer boundaries was first demonstrated in 2010 when Vierbuchen et al. converted mouse fibroblasts into neuron-like cells by forced expression of a small set of neurogenic transcription factors (TFs) [5]. The idea of inducing cell-fate changes in vitro dates back to the 1980s when

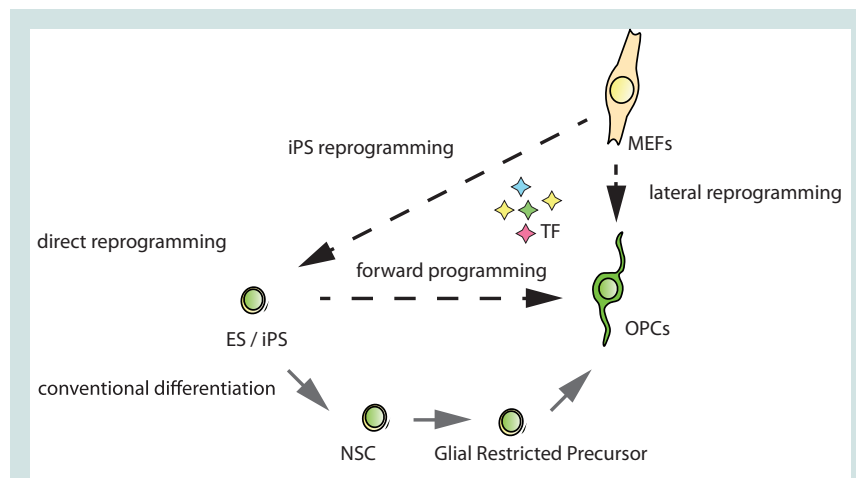

Figure 1: Direct cellular reprogramming differs from conventional differentiation in that it is initiated by forced expression / silencing of reprogramming factors and that it is direct and does not entail a stepwise progression of known precursor stages to reach the targeted cell population. 
a series of pioneering studies conducted by Davis, Weintraub, and Lassard showed that forced expression of the TF Myod 1 in fibroblasts and other mesoderm-derived cells was sufficient to convert the transfected cells into cells with myoblast-like features [6]. These findings not only emphasized the pivotal role of TFs in the control of cell specific transcriptional networks, they also indicated that fullydifferentiated cellular entities may be regarded as metastable states, which are less cemented in their identity than initially thought and which remain amenable to cell fate engineering. However, when Myod1 was expressed in cells with endo- or ectodermal origin, only a partial conversion was observed. Despite the successful induction of skeletal-muscle specific programs, the characteristic transcriptional network of the starting cells remained expressed and was only incompletely silenced, indicating either that germ-layer boundaries hold firm or that additional cues are necessary to induce a full lineage conversion across germ-layer boundaries. Two decades later, the direct conversion of murine fibroblasts into functional neurons by overexpression of only three TFs demonstrated a new and fast route from fibroblasts to neurons and provided proof-of-principle for lineage conversions across germ layers [5].

\section{Induced Neuronal Cells}

Studies investigating the possibilities of directly converting cells into neurons can be broadly divided into two categories. The first group of studies aims to provide proof-of-principle of successful conversion of various starting cell types into cells that display generic traits of neurons or to investigate the underlying mechanisms. On the other hand the aim of the second group of studies is to refine the reprogramming protocols to generate cell-types that resemble their respective in vivo counterparts as closely as possible [7].

\section{Induced neuronal cells}

The neuron-like cells obtained by the first reported trans-lineage conversion of mouse embryonic fibroblasts (MEFs) were characterised by the expression of pan-neuronal markers, their ability to fire action potentials and to form synapses [5]. Neuronal conversion of MEFs was initiated by over overexpression of three TFs (Ascl1, Brn2, Myt1l; collectively referred to as BAM, (Figure 2a)), which were chosen from an initial 19-factor pool using an empiric approach. Notably, the proneural gene Ascll alone suffices to convert MEFs into cells with an immature neuronal morphology and expression of some neuronal markers. The addition of Brn2 and Myt1l rendered the conversion
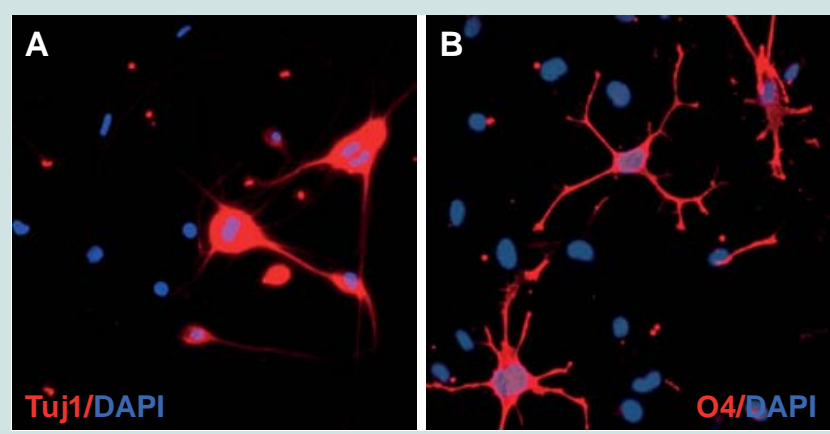

Figure 2: a) MEFs reprogrammed into induced Neurons (iN) with BAM (Vierbuchen et al., 2010) and stained with Tuj1 15 days after induction. b) MEFs induced into oligodendrocyte precursor cells (iOPCs) stained for O4 and DAPI 15 days post induction. process more efficient and, importantly, conferred further neuronal characteristics and in particular electrophysiological properties to the induced cells. In a follow-up study, the group went on to demonstrate that the same TF-combination is sufficient to convert primary mouse hepatocytes into neuronal cells, so providing proof-of-concept for the feasibility of endoderm-to-ectoderm conversions at least in rodent cells [8]. Both studies used lentiviral vectors for stable and sustained expression of the exogenous transgenes. However, alternative nonintegrative gene-delivery methods have also been successfully applied in reprogramming experiments with murine fibroblasts, including adenoviral gene-delivery (Ascl1, Brn2, Ngn2) [9] and repeated plasmid-based gene-delivery (BAM-factors) mediated by bioreducible linear poly(amido amine)s [10].

Human fibroblasts have also been successfully converted into functional neurons by over expressing the BAM-set of TFs, albeit with lower efficiency. The resulting neurons were less mature when compared to rodent induced neurons [11,12]. Subsequent investigations aimed to enhance the efficiency of the conversion from human fibroblasts into neurons. Notable improvements were achieved by adding Neurod1 [11] or Zic1 [13] to the basic BAMrecipe. Interestingly, one study demonstrated that replacing the factors Brn2 and Myt1l of the BAM-pool with Ngn2 resulted in more efficient reprogramming and that the addition of small molecules was able to enhance neuronal yield and purity further [14]. These findings and data of the study using adenoviral constructs [9] suggest that reprogramming using the two-TF combination Ascll and Ngn2 may be superior to the use of the BAM-set with respect to the reprogramming efficiency measured by the total number of $\beta I I I-t u b u l i n$ positive cells. Another study demonstrated that lowering the $\mathrm{O}_{2}$ tension at the initiation of reprogramming from atmospheric (21\%) to physiological (5\%) levels increases the overall neuronal yield and also results in more functional induced neurons [15].

In addition to TFs microRNAs (miRs) have also proven to be potent reprogramming factors. Functional neurons have been generated by over overexpression of miR-124 in combination with Brn2 and Myt1l (thus replacing the pioneering TF Ascl1 [16] or by combining miR-124 with miR-9/9* and Neurod2 [17]). The addition of the TFs Ascl1 and Brn2 to the miR combination resulted in further improvements.

More recently a new reprogramming strategy which does not rely on forced expression of TFs or miRs was presented [18]. Xue et al. demonstrated that the knock-down of a single RNAbinding polypyrimidine tract-binding (PTB) protein is sufficient to convert MEFs into neuronal cells. The mechanism underlying the reprogramming process is the release of a PTB-mediated blockade of miR-activity on several components of the REST (RE1-silencing transcription factor) complex and the resulting de-repression of neuron-specific genes.

Finally, one study has provided proof-of-concept that induced neuronal cells (Ascl1, Brn2, Myt1l, Zic1) from patients suffering of Alzheimer's disease faithfully reproduce certain aspects of the disease and thus can be applied for the study of mechanisms underlying neurodegenerative disorders [13]. In this study induced neurons from patients with genetic forms of Alzheimer's disease were compared to wild-type controls with regard to their processing of amyloid precursor protein (APP) and the formation of amyloid (A) $\beta$. Patientderived cells that carry a mutation in the presenilin 1 (PSEN1)-gene 
show an increased $A \beta_{42}: A \beta_{40}$ ratio, a hallmark of the three major autosomal-dominant forms of Alzheimer's disease.

\section{Induced dopaminergic neurons}

For many applications the generation of a well-defined subset of neurons is preferable. A good example is mesencephalic dopaminergic neurons, whose loss is considered to be a pathological hallmark of Parkinson's disease. Numerous protocols exist for the differentiation of human PSCs into dopaminergic neurons and allow modelling of certain aspects of monogenetic forms of Parkinson's disease. Similarly, in the young field of direct reprogramming, a handful of protocols have been published for the direct conversion of fibroblasts into dopaminergic neurons. An important question that remains to be answered is whether the protocols, which differ with respect to the combination of reprogramming factors used, ultimately produce the same cell type or whether they produce cells, which overall are similar but differ in individual cellular traits. Do the individual dopaminergic neuronal phenotypes produced by the various protocols represent different aspects of the same in vivo cell type with different levels of fidelity? Or does one particular protocol produce cells that resemble their in vivo counterparts closer than the cells derived by other protocols?

The first induced dopaminergic neurons were generated from human fetal fibroblasts by overexpressing the neurogenic BAM set in combination with two additional TFs specific for the dopaminergic lineage (Lmx1a, Foxa2) [19]. Subsequently, it was demonstrated that a limited set of three TFs (Ascl1, Nurr1, Lmxla) is also capable to induce dopaminergic neurons from both mouse embryonic and adult human fibroblasts. This new reprogramming set seems to rely on similar principles as the previous approach: the combination of classical neurogenic TFs (e.g. Ascl1, Brn2, Myt1l) with further, lineage specific TFs (e.g. Foxa2, Lmx1a, Nurr1) [20]. In both studies, the resulting cells displayed morphological and electrophysiological features characteristic of dopaminergic neurons. However, the gene expression profiles of the reprogrammed dopaminergic neurons differed significantly from primary midbrain dopaminergic neurons. In yet another study Ascl1 and Brn2 were combined with various combinations of five lineage specific TFs (Lmxla, Lmx1b, Foxa2, Nurr1, Otx2) [21]. However, the experiments were limited to MEFs and the reported conversion efficiencies were low and detailed analysis of the induced dopaminergic neurons by profiling or an in vivo evaluation was not conducted. Another group has generated cells resembling dopaminergic neurons from mouse tail tip fibroblasts by overexpression of six TFs (Ascl1, En1, Foxa2, Lmx1a, Nurr1, Pitx3) [22]. In addition, the morphogen sonic hedgehog and FGF8 were added to the culture medium. The so generated induced dopaminergic neurons again resembled the typical morphology of dopaminergic neurons and showed appropriate transmitter release. However, in this study only murine fibroblasts were used and the induced neuronal cells differed significantly from primary mesencephalic dopaminergic neurons with respect to their transcriptional profile. Nevertheless, transplantation of the cells into the striatum of mice with 6-hydroxydopamine (6-OHDA)-induced unilateral destruction of the nigrostriatal dopamine system resulted in a partial but significant rescue-effect of amphetamine-induced rotation scores. The newest protocol for the direct conversion of human fibroblasts into dopaminergic neurons involves forced expression of five TFs (Ascl1, Ngn2, Nurr1, Pitx3, Sox2) [23]. The induced dopaminergic neurons obtained by this combination appear to be more morphologically mature when compared to the previous combinations. Grafting of the induced neurons into 6-OHDA-lesioned rats led to a significant stabilisation of the animals' amphetamine-induced rotational movements one and two months following cell transplantation. Unfortunately, the species differences and the distinct experimental setups do not allow for a direct comparison of the two reprogramming protocols for which functional in vivo studies have been conducted.

\section{Induced cholinergic neurons}

Another neuronal cell population that has attracted particular interest are cholinergic motoneurons. Loss of cholinergic motoneurons is the main pathological feature of amyotrophic lateral sclerosis. In one study, mouse and human fibroblasts were successfully reprogrammed by simultaneous forced expression of 8TFs: Ascl1, Brn2, Myt1l, Neurod1, Ngn2, Lhx3, Hb9, Isl1 [24]. Co-transduction of the 8-TF set resulted in induced cells resembling motoneurons. Functional studies demonstrated that the induced motor neurons displayed typical electrophysiological properties and engage in functional synapse formation with myotubes in vitro. Grafting of induced motor neurons into the developing chick spinal cord, showed that the majority of the cells integrated into the appropriate ventral horn-related circuits. However, the reprogramming efficiency of human fibroblasts was very low restricting the use of the cells for further biomedical applications.

\section{Induced Glia}

More than three years following the first report of successfully induced neuronal cells [5], two independent groups demonstrated the conversion of murine fibroblasts into functional oligodendrocyte precursors [25,26] (Figure 2b). The core component of both reprogramming TF sets consisted of Olig2 and Sox10, both TFs that play essential roles during oligodendrocyte development. In one study this combination was supplemented with Znf536 [25], in the other with Nkx6.2 [26]. Conversion efficiencies in both studies were low. However, the proliferative capacity (even though limited) of the induced OPCs mitigated this problem to some extent. Induced OPCs were characterised by typical morphological features and marker expression. The gene expression profiles resembled to some extent those of primary OPCs. A minority of purified induced OPCs differentiated into mature myelinating oligodendrocytes and gave rise to myelin sheaths when co-cultured with DRG-neurons in vitro. Furthermore, Olig2/Sox10/Znf536 induced $\mathrm{O}^{+}$rat-OPCs were purified and transplanted into the corpus callosum and the cerebellum of neonatal shiverer mice. Three months following the grafting of the cells small clusters of mature myelinating oligodendrocytes were found at all injection sites [25].

\section{Induced Neural Stem Cells}

One of the major limitations of direct lineage reprogramming approaches applied to the generation of neurons and glia is their restricted scalability. Neurons, for example, are post-mitotic and cannot be expanded to reach the numbers required for many downstream applications. Especially for applications that require large cell quantities, such as cell transplantation treatments or highthroughput screening, a more indirect approach may be advantageous. As a step towards a renewable source of induced neural cells protocols were developed for direct conversion of mouse fibroblasts into selfrenewing and multipotent neural stem cells (NSCs). Induced tripotent NSCs could serve as starting cells for differentiation into the principle 
cell types of the CNS, instead of neurons, astrocytes, and oligodendrocytes. Two fundamentally different approaches have so far been applied to the generation of induced NSCs from fibroblasts. The first consists in a transient (3-6 days) expression of the four pluripotency factors (Oct4, Sox2, Klf4, c-Myc) followed by an extended culture period in neural induction medium [27]. Rosetteforming colonies that exhibited a NSC-like morphology and characteristic marker expression appeared with a low efficiency that was comparable to iPSC-induction. The induced NSCs successfully differentiated into neurons and astrocytes but oligodendrocyte differentiation was not reported. The ability of the induced NSCs to be passaged seemed also compromised. From a mechanistic perspective it would be very interesting to know to which extent the $0.5 \%$ of cells that successfully converted to NSCs was reprogrammed towards pluripotency prior to NSC-formation. A different approach for the generation of iNSCs consisted in the transient expression of the pluripotency factor Oct4 (for five days) while maintaining a continuous expression of Sox2, Klf4 and c-Myc [28]. It was hypothesized that pluripotent intermediates that were generated under the influence of Oct 4 were re-directed by Sox 2 towards the neural lineage before acquiring full pluripotency. Although the reprogramming efficiency reported was very low, the induced NSCs were self-renewable and could be expanded for more than 50 passages, in principle allowing for the generation of sufficient cells quantities. Furthermore, the authors demonstrated the ability of the cells to generate all three cell types, neurons, oligodendrocytes and astrocytes both in vitro and in vivo.

Two other groups sought to induce NSCs based on empiric selection of more than ten lineage-specific candidate TFs. Lujan et al. generated induced NSCs by overexpressing Sox2, Brn2, and FoxG1 [29]. Han et al. found a 4-factor combination consisting of Sox2, Brn4, Klf4, and c-Myc most efficient [30]. Both protocols do not transit via pluripotent intermediates and yield tripotent, selfrenewable NSCs. However, long derivation times (several weeks) and low conversion efficiencies $(<0.5 \%)$ may mitigate potential advantages of this approach. More recently the generation of NSCs from MEFs and human fetal fibroblasts was achieved by forced expression of Sox 2 alone [31].

It remains to be seen whether the differentiation of induced NSCs into mature neural cells can stand up to the more established and more standardised iPSC-based protocols.

\section{Technical Issues}

A number of important technical hurdles need to be overcome before direct cellular reprogramming can be used for generating sufficient quantities of purified human neural cells. These include the relatively low conversion efficiencies, the heterogeneity of the induced cells, even following purification, the limited scalability and further practical limitations.

\section{Conversion efficiency and scalability}

Many biomedical applications for which induced neural cells may be used require large numbers of cells. However, the generation of post-mitotic neural cell-types by existing direct cellular reprogramming protocols of somatic cell types is still relatively inefficient. Although cells can convert remarkably fast from one cell type to another, the absence of a proliferative stage compromises the scalability of the system. Landmark studies suggest that the direct conversion of cells into somatic cells not only bypasses pluripotent intermediates but also avoids transitioning through a proliferative precursor state [32]. In the absence of a proliferative step the number of post-mitotic induced-cells that can be obtained is finite and depends on three variables: the number of starting cells, their capacity to undergo the conversion process, and the efficiency inherent to any given reprogramming protocol.

\section{The source cell type}

The starting cell population used for most reprogramming experiments consists of different types of fibroblasts. Fibroblasts are a morphologically heterogeneous group of mesenchymal cells, which are found abundantly in connective tissues. Patient-derived fibroblasts are easy to obtain, easy to culture and they are relatively amenable to cell-fate engineering. But are they also the best cell-type for neural reprogramming? Following their successful conversion of mouse hepatocytes into neurons, Marro et al. have raised the question which the ideal starting cell-population for a given target cell of interest would be [8]. It is not surprising that more closely related cells such as astrocytes or pericytes undergo direct conversion into neurons with simpler sets of reprogramming-factors than those used for the conversion of fibroblasts $[33,34]$. This is an important finding that may have major implications for in vivo reprogramming and in vivo cell-replacement strategies that aim at promoting repair [35]. However, as human neuroectoderm-derivatives are difficult to obtain potential advantages with respect to their capability of being reprogrammed into neural cells cannot be applied for the in vitro manufacture of such cells. Ladewig et al. highlighted the potential of umbilical cord blood-derived stem cells (CB-SC) as an alternative cell source that is relatively free of age-related mutations and available from numerous cell banks [14]. The reported conversion efficiencies of CB-SCs were higher than those of adult fibroblasts but lower than those of postnatal fibroblasts. So far other more available cell types such as keratinocytes or peripheral blood cells have not yet been tested for their capability of generating induced neural cells. Despite the requirement for more elaborate cell culture systems keratinocytes would be interesting candidates because of the ectodermal origin, which they share with neural cells.

\section{The gene-delivery method}

The potency of the reprogramming-protocol is a further critical determinant for the yield of target cells. Most studies so far are based on co-transduction of multiple lentiviral vectors. This approach served as the central technology in virtually all reprogramming studies so far. However, the approach can also be regarded as one of the selflimiting factors that restrict progress of cellular reprogramming with respect to cell yield and purity. Even under optimised conditions the transduction efficiency for a single viral vector does not reach $100 \%$, but ranges from $30-95 \%$ in studies that reprogram fibroblasts into various cell types $[24,26]$. The proportion of cells co-expressing all TFs of a given TF-set can be roughly calculated from the transduction efficiency of a single vector. In a fictitious example, in which cells are reprogrammed by forced expression of five viral vectors, each with an average transduction efficiency of $70 \%$, only $(0.7)^{5} \times 100 \%=$ $12 \%$ of the cells were to co-express the entire set of reprogramming factors. Therefore, in the absence of any redundancy amongst the reprogramming factors only $12 \%$ of cells would have the chance to convert into the desired target cell. This calculation suggests that the efficiencies of some published protocols already reached the limits intrinsic to this particular approach. In reality, however, the co- 
transduction problem is mitigated by the fact that viral vectors have a tendency to co-transduce the same cells. Nevertheless, it is useful to bear this calculation in mind when evaluating reprogramming efficiencies. As a first step to address this issue polycistronic gene delivery systems have been developed [13], which may also resolve issues related to stoichiometric differences in the expression of individual factors in the host cell.

\section{Heterogeneity of the reprogrammed cell population}

Ladewig et al. have stressed the importance of reporting yield and purity as two separate entities when determining the success of a new reprogramming strategy [14]. Current reprogramming techniques invariably lead to very heterogeneous cell-populations, which contain a mixture of different cell types. These range from fully reprogrammed cells closely resembling the population of interest, to partially reprogrammed, or possibly remaining cells which did not undergo reprogramming. For most downstream applications all partially reprogrammed cells must be regarded as contaminating cells and further purification will be necessary. However, especially mature neurons are fragile and their survival rate after passaging and additional purification steps is low [36]. Moreover, cell-sorting using established cell surface markers may not be able to differentiate subtle differences between partially and fully reprogrammed cells.

Further heterogeneity of induced cells may occur as a consequence of the gene-delivery method used for the expression of the TF set. With only few exceptions, lentiviral vectors have been used to integrate the reprogramming-factors stably into the genome and to achieve robust transgene expression. Fibroblasts do not allow for single-cell clonal expansion, and therefore the copy number of the integrated provirus and the random genomic integration invariably will result in cell populations that are highly heterogeneous from a genomic standpoint. This problem is further complicated by the cotransduction of multiple reprogramming factors, which also raises the question of the influence of reprogramming factor stoichiometry in the process of cellular reprogramming [37].

\section{Future Perspectives}

Direct cellular reprogramming has attracted a large and growing scientific community in a short period of time and opened the ways for previously unthought-of lines of research. Progress in several aspects of lineage reprogramming is now eagerly awaited.

Technical improvements to overcome the hurdles outlined in the previous sections are a prerequisite for many biomedical applications. Other non-integrative gene-delivery methods such as synthetic mRNAs or Sendai-virus or PiggyBac, as it was shown for the relatively efficient generation of iPSCs [38-40], may represent an alternative to circumvent some of the drawbacks and risks associated with integrative viral gene delivery. Precise recruitment of inhibition of signalling pathways by small molecules may facilitate distinct reprogramming pathways and even lead to functional substitution of individual TFs. Until the "perfect" reprogramming strategy exists, the most suitable approach may vary depending on the research purpose that will determine whether large quantities, high levels of cellular purity and homogeneity, or the closest resemblance to an in vivo cellular subtype are the predominant requirement.

Direct conversion of fibroblasts into tripotent neural stem cells addresses some of the issue of limited scalability of reprogrammingbased strategies for post-mitotic target cells. Neural progenitors may be expanded to generate the final numbers required for biomedical applications. Similarly, induced OPCs are expandable, at least for a few passages, mitigating the scalability problem [26]. However, with only one notable exception [31], so far reprogramming-based protocols for the direct conversion of fibroblasts into expandable progenitor cells are only available for rodent cells. Similar to iPSCbased strategies the generation of the target cell of interest from NSCs will require differentiation steps with their intrinsic limitations. In the future a direct comparison of iPSC and iNSC-derived neurons or glia with their respective differentiation protocols will be necessary.

The reprogramming process itself and its cellular consequences need to be explored in detail. Direct reprogramming is associated with dramatic genome-wide transcriptional and epigenetic changes. However, many of the underlying mechanisms, how these changes can be induced by just a small number of TFs remain obscure. It has been hypothesised, that some of the TFs used in reprogramming experiments serve as pioneers that are capable of activating the expression of their target genes even in a repressed chromatin state [3-5,41]. The resulting combination of exogenous reprogramming factors and activated endogenous TFs are sufficient to silence the transcriptional programs of the starting cell and to activate those of the target cell. However, evidence from the related iPSCresearch suggests that even target cells that are considered to be fully reprogrammed retain epigenetic marks, which the corresponding donor cells had progressively acquired undergoing developmental differentiation [42]. Their significance and their impact on the target cell are currently a matter of debate.

Another unresolved question concerns the age of donor cells. Tissue that serves as starting material for modelling or treating the major neurodegenerative diseases is typically derived from elderly patients. The influence of the donor's age on cell reprogramming is still unclear. Some authors observed no decrease in the conversion efficiency in cells derived from aged individuals [12] while others showed an inverse correlation between the donor's age and the conversion efficiency [14]. Furthermore, it is unknown whether and to which extent the biological age of the starting cell is conserved in the target cell. Does a 60 year old fibroblast convert into a 60 year old neuron or are certain cell characteristics reset to an earlier developmental stage? One of the molecular mechanisms underlying cellular aging is the progressive shortening of telomeres [43]. Interestingly, telomerase activity in iPSCs derived from somatic adult tissue is increased. In fact, iPS induction is capable of fully restoring telomere length [44], but what happens to the telomeres if one somatic cell is directly converted into another?

Finally, the refinement and development of new reprogramming protocols will be an important future aim. Differences in the genomic expression of human induced dopaminergic or glutamatergic neurons suggest the need of further improvements. Furthermore, numerous other neuronal subtypes await to be programmed. From the perspective of clinical cell transplantation, the generation of oligodendrocyte precursors is perhaps the most exciting development so far. Amongst the group of neurodegenerative disease, classical genetic demyelinating diseases are arguably the ones that are most likely to profit from cell replacement therapies [45]. As the generation of human oligodendrocytes from pluripotent stem cells by cell differentiation is still particularly cumbersome [46], the development of direct reprogramming strategies into human oligodendrocytes is highly desirable. The recent studies on the successful conversion of 
rodent fibroblasts into oligodendrocyte precursors are a first step. Translation of these findings to human cells is necessary. Furthermore, the road blocks in the way of low reprogramming efficiency need to be identified and removed. Robust culture systems for human neural cells would mark milestones in the neurosciences and unfold exciting new avenues of translational research [47].

\section{References}

1. Thomson JA, Itskovitz-Eldor J, Shapiro SS, Waknitz MA, Swiergiel JJ, et al. (1998) Embryonic stem cell lines derived from human blastocysts. Science 282: 1145-1147.

2. Takahashi K, Tanabe K, Ohnuki M, Narita M, Ichisaka T, et al. (2007) Induction of pluripotent stem cells from adult human fibroblasts by defined factors. Cell 131: 861-872.

3. Vierbuchen T, Wernig M (2011) Direct lineage conversions: unnatural but useful? Nat Biotechnol 29: 892-907.

4. Ladewig J, Koch P, Brüstle O (2013) Leveling Waddington: the emergence of direct programming and the loss of cell fate hierarchies. Nat Rev Mol Cell Biol 14: $225-236$.

5. Vierbuchen T, Ostermeier A, Pang ZP, Kokubu Y, Südhof TC, et al. (2010) Direct conversion of fibroblasts to functional neurons by defined factors. Nature 463: 1035-1041.

6. Davis RL, Weintraub $H$, Lassar AB (1987) Expression of a single transfected cDNA converts fibroblasts to myoblasts. Cell 51: 987-1000.

7. Yang N, Ng YH, Pang ZP, Südhof TC, Wernig M (2011) Induced neuronal cells: how to make and define a neuron. Cell Stem Cell 9: 517-525.

8. Marro S, Pang ZP, Yang N, Tsai MC, Qu K, et al. (2011) Direct lineage conversion of terminally differentiated hepatocytes to functional neurons. Cell Stem Cell 9: 374-382

9. Meng F, Chen S, Miao Q, Zhou K, Lao Q, et al. (2012) Induction of fibroblasts to neurons through adenoviral gene delivery. Cell Res 22: 436-440.

10. Adler AF, Grigsby CL, Kulangara K, Wang H, Yasuda R, et al. (2012) Nonvira direct conversion of primary mouse embryonic fibroblasts to neuronal cells. Mol Ther Nucleic Acids 1: e32.

11. Pang ZP, Yang N, Vierbuchen T, Ostermeier A, Fuentes DR, et al. (2011) Induction of human neuronal cells by defined transcription factors. Nature 476: 220-223.

12. Pfisterer U, Wood J, Nihlberg K, Hallgren O, Bjermer L, et al. (2011) Efficient induction of functional neurons from adult human fibroblasts. Cell Cycle 10: 3311-3316.

13. Qiang L, Fujita R, Yamashita T, Angulo S, Rhinn H, et al. (2011) Directed conversion of Alzheimer's disease patient skin fibroblasts into functional neurons. Cell 146: 359-371.

14. Ladewig J, Mertens J, Kesavan J, Doerr J, Poppe D, et al. (2012) Small molecules enable highly efficient neuronal conversion of human fibroblasts. Nat Methods 9: 575-578.

15. Davila J, Chanda S, Ang CE, Südhof TC, Wernig M (2013) Acute reduction of oxygen tension enhances the induction of neurons from human fibroblasts. $J$ Neurosci Methods 216: 104-109.

16. Ambasudhan R, Talantova M, Coleman R, Yuan X, Zhu S, et al. (2011) Direct reprogramming of adult human fibroblasts to functional neurons under defined conditions. Cell Stem Cell 9: 113-118.

17. Yoo AS, Sun AX, Li L, Shcheglovitov A, Portmann T, et al. (2011) MicroRNAmediated conversion of human fibroblasts to neurons. Nature 476: 228-231.

18. Xue Y, Ouyang K, Huang J, Zhou Y, Ouyang H, et al. (2013) Direct conversion of fibroblasts to neurons by reprogramming PTB-regulated microRNA circuits. Cell 152: 82-96.

19. Pfisterer U, Kirkeby A, Torper O, Wood J, Nelander J, et al. (2011) Direct conversion of human fibroblasts to dopaminergic neurons. Proc Natl Acad Sci
U S A 108: 10343-10348.

20. Caiazzo M, Dell'Anno MT, Dvoretskova E, Lazarevic D, Taverna S, et al. (2011) Direct generation of functional dopaminergic neurons from mouse and human fibroblasts. Nature 476: 224-227.

21. Sheng C, Zheng Q, Wu J, Xu Z, Sang L, et al. (2012) Generation of dopaminergic neurons directly from mouse fibroblasts and fibroblast-derived neural progenitors. Cell Res 22: 769-772.

22. Kim J, Su SC, Wang H, Cheng AW, Cassady JP, et al. (2011) Functiona integration of dopaminergic neurons directly converted from mouse fibroblasts. Cell Stem Cell 9: 413-419.

23. Liu X, Li F, Stubblefield EA, Blanchard B, Richards TL, et al. (2012) Direct reprogramming of human fibroblasts into dopaminergic neuron-like cells. Cell Res 22: 321-332.

24. Son EY, Ichida JK, Wainger BJ, Toma JS, Rafuse VF, et al. (2011) Conversion of mouse and human fibroblasts into functional spinal motor neurons. Cel Stem Cell 9: 205-218.

25. Yang N, Zuchero JB, Ahlenius H, Marro S, Ng YH, et al. (2013) Generation of oligodendroglial cells by direct lineage conversion. Nat Biotechnol 31: 434439.

26. Najm FJ, Lager AM, Zaremba A, Wyatt K, Caprariello AV, et al. (2013) Transcription factor-mediated reprogramming of fibroblasts to expandable, myelinogenic oligodendrocyte progenitor cells. Nat Biotechnol 31: 426-433.

27. Kim J, Efe JA, Zhu S, Talantova M, Yuan X, et al. (2011) Direct reprogramming of mouse fibroblasts to neural progenitors. Proc Natl Acad Sci U S A 108: 7838-7843.

28. Thier M, Wörsdörfer P, Lakes YB, Gorris R, Herms S, et al. (2012) Direct conversion of fibroblasts into stably expandable neural stem cells. Cell Stem Cell 10: 473-479.

29. Lujan E, Chanda S, Ahlenius H, Südhof TC, Wernig M (2012) Direct conversion of mouse fibroblasts to self-renewing, tripotent neural precursor cells. Proc Natl Acad Sci U S A 109: 2527-2532.

30. Han DW, Tapia N, Hermann A, Hemmer K, Höing S, et al. (2012) Direct reprogramming of fibroblasts into neural stem cells by defined factors. Cel Stem Cell 10: 465-472.

31. Ring KL, Tong LM, Balestra ME, Javier R, Andrews-Zwilling Y, et al. (2012) Direct reprogramming of mouse and human fibroblasts into multipotent neural stem cells with a single factor. Cell Stem Cell 11: 100-109.

32. leda M, Fu JD, Delgado-Olguin P, Vedantham V, Hayashi Y, et al. (2010) Direct reprogramming of fibroblasts into functional cardiomyocytes by defined factors. Cell 142: 375-386.

33. Heinrich C, Blum R, Gascón S, Masserdotti G, Tripathi P, et al. (2010) Directing astroglia from the cerebral cortex into subtype specific functiona neurons. PLoS Biol 8: e1000373.

34. Karow M, Sánchez R, Schichor C, Masserdotti G, Ortega F, et al. (2012) Reprogramming of pericyte-derived cells of the adult human brain into induced neuronal cells. Cell Stem Cell 11: 471-476.

35. Rouaux C, Bhai S, Arlotta P (2012) Programming and reprogramming neuronal subtypes in the central nervous system. Dev Neurobiol 72: 10851098.

36. Shi Y, Kirwan P, Livesey FJ (2012) Directed differentiation of human pluripotent stem cells to cerebral cortex neurons and neural networks. Nat Protoc 7: 1836-1846.

37. Carey BW, Markoulaki S, Hanna JH, Faddah DA, Buganim Y, et al. (2011) Reprogramming factor stoichiometry influences the epigenetic state and biological properties of induced pluripotent stem cells. Cell Stem Cell 9: 588598.

38. Zhou H, Wu S, Joo JY, Zhu S, Han DW, et al. (2009) Generation of induced pluripotent stem cells using recombinant proteins. Cell Stem Cell 4: 381-384.

39. Fusaki N, Ban H, Nishiyama A, Saeki K, Hasegawa M (2009) Efficient induction of transgene-free human pluripotent stem cells using a vector 
Citation: Pawlowski M, Kotter MR. Generation of Neural Cells by Direct Cellular Reprogramming. J Transplant Stem Cel Biol. 2013;1(1): 7.

ISSN: 2374-9326

based on Sendai virus, an RNA virus that does not integrate into the host genome. Proc Jpn Acad Ser B 85: 348-362.

40. Woltjen K, Michael IP, Mohseni P, Desai R, Mileikovsky M, et al. (2009) piggyBac transposition reprograms fibroblasts to induced pluripotent stem cells. Nature 458: 766-770.

41. Cirillo LA, Lin FR, Cuesta I, Friedman D, Jarnik M, et al. (2002) Opening of compacted chromatin by early developmental transcription factors HNF3 (FOXA) and GATA-4. Mol Cell 9: 279-289.

42. Kim K, Doi A, Wen B, Ng K, Zhao R, et al. (2010) Epigenetic memory in induced pluripotent stem cells. Nature 467: 285-290.

43. Blasco MA (2005) Telomeres and human disease: ageing, cancer and beyond. Nat Rev Genet 6: 611-622.
44. Marion RM, Strati K, Li H, Tejera A, Schoeftner S, et al. (2009) Telomeres acquire embryonic stem cell characteristics in induced pluripotent stem cells. Cell Stem Cell 4: 141-154.

45. Franklin RJ, Ffrench-Constant C (2008) Remyelination in the CNS: from biology to therapy. Nat Rev Neurosci 9: 839-855.

46. Wang S, Bates J, Li X, Schanz S, Chandler-Militello D, et al. (2013) Human iPSC-derived oligodendrocyte progenitor cells can myelinate and rescue a mouse model of congenital hypomyelination. Cell Stem Cell 12: 252-264.

47. Goldman SA, Nedergaard M, Windrem MS (2012) Glial progenitor cell-based treatment and modeling of neurological disease. Science 338: 491-495.

\section{Acknowledgements}

Dr. Matthias Pawlowski is supported by a stipend from the German Research Foundation (DFG; PA 2369/1-1). 